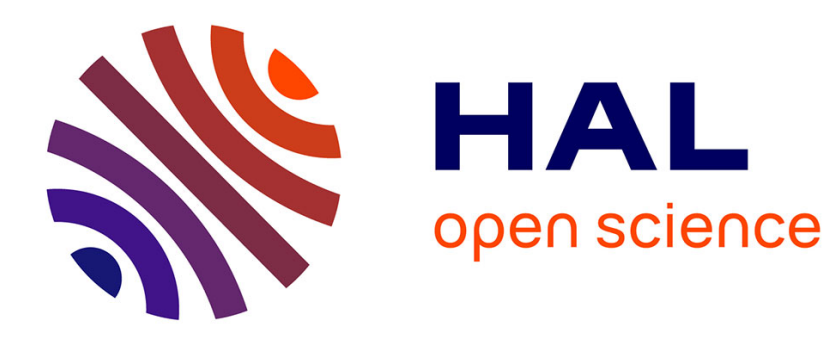

\title{
Marchands et philanthropes Les associations de bienfaisance chinoises en Thaïlande
}

Bernard Formoso

\section{To cite this version:}

Bernard Formoso. Marchands et philanthropes Les associations de bienfaisance chinoises en Thaïlande. Annales. Histoire, Sciences sociales, 2003, 4, pp.833 - 856 . hal-01388086

\section{HAL Id: hal-01388086 \\ https://hal.science/hal-01388086}

Submitted on 26 Oct 2016

HAL is a multi-disciplinary open access archive for the deposit and dissemination of scientific research documents, whether they are published or not. The documents may come from teaching and research institutions in France or abroad, or from public or private research centers.
L'archive ouverte pluridisciplinaire $\mathbf{H A L}$, est destinée au dépôt et à la diffusion de documents scientifiques de niveau recherche, publiés ou non, émanant des établissements d'enseignement et de recherche français ou étrangers, des laboratoires publics ou privés. 


\title{
Marchands et philanthropes Les associations de bienfaisance chinoises en Thaïlande
}

\author{
Bernard Formoso
}

Les institutions examinées dans les pages suivantes sont appelées hsiang t’üng (Maison de bienfaisance dans la langue teochiu ${ }^{1}$ ) de la région de Chaozhou (environs de la ville de Shantou, province de Guangdong), qui fut le principal foyer d'émigration des Hua Ch'iao (Chinois d'outre-mer) établis en Thaïlande. Hsiang t'üng correspond au mandarin shan tang, tandis qu'en langue thaïe, seul idiome que la plupart des immigrés chinois de la seconde ou de la troisième génération connaissent, le terme couramment employé est celui de munithi qui signifie «association philanthropique».

Ces formes d'organisation jouent un rôle de premier plan dans le maintien d'une tradition liturgique chinoise, ainsi que d'une identité cultuelle proprement teochiu dans le contexte thaïlandais. Leur transposition au Siam s'est d'autant mieux réalisée que les shan tang perpétuaient des fonctions sociales, politiques et humanitaires qui avaient fait leur succès dans la Chine de la seconde moitié du $\mathrm{XIX}^{\mathrm{e}}$ siècle. Elles poursuivirent ainsi les missions d'assistance aux victimes de désastres et d'inhumation des pauvres ou des morts sans descendance qui avaient été leur vocation première dans la mère patrie. Néanmoins, il n’y eut pas simple translation. Ces associations acquirent une nouvelle dimension tant du point de vue culturel qu'organisationnel. Du fait de la politique anti-chinoise que les nationalistes thaïs imposèrent dans les années 1930-1950, elles concentrèrent, par la

1 - Le système de transcription des termes chinois pour lequel j'ai opté est dérivé de celui de Wade et a été adapté à la spécificité de la langue teochiu (/p\%,/t'/, etc., sont des consonnes éjectives). 
plus grande tolérance que leurs activités philanthropiques suscitaient, différentes fonctions religieuses qu'un tissu plus diversifié d'associations ethniques assurait auparavant. Enfin, et ce n'est pas le moindre de leur intérêt, l'histoire des lignées qu'elles forment souvent reflète parfaitement le processus d'expansion dans l'espace et l'essor économique de la communauté chinoise locale.

Dans les villes où elles sont établies, leurs temples sont devenus en effet le lieu de célébration des grandes fêtes calendaires, et les mieux organisées d'entre elles ont des équipes de prêtres qui supervisent, à la demande, la conduite des rites funéraires que des associations claniques ou bouddhistes plus spécialisées prenaient jadis en charge. Elles sont aussi, pour la plupart, les promotrices d'une intense activité médiumnique tendue vers la délivrance d'oracles et de moyens prophylactiques ou thérapeutiques. Enfin, par transposition outre-mer de certains cultes teochiu, elles sous-tendent l'identité de ce groupe tout en contribuant à l'intégration socio-politique de la minorité chinoise à l'ensemble national thaïlandais. Cette dernière fonction, elles l'assument par leurs activités caritatives. Une majorité d'entre elles dispose en effet de moyens de secours (ambulances, équipes de sauveteurs, voire hôpitaux) qui assistent les pouvoirs publics en cas d'accidents de circulation ou de désastres majeurs (incendies, inondations, déraillements de train, etc.). À cela s'ajoute la fourniture de biens de première nécessité et, parfois, la délivrance de soins gratuits aux plus pauvres. Enfin, leur plus grand sujet de fierté est la prise en charge des morts sans descendance qu'elles accueillent dans leurs cimetières, qu'elles expédient dans l'Au-delà par le biais d'une fête décennale (par le rituel du hsiu kou ku) et auxquels elles vouent ensuite un culte en tant que famille adoptive. Par ces actes largement médiatisés, les shan tang donnent des gages ostentatoires de sens civique dont profitent plus ou moins directement l'ensemble des Hua Ch'iao du pays.

\section{Maisons de bienfaisance et mouvement sectaire}

Par rapport aux shan tang instituées dans d'autres pays d'Asie du Sud-Est, celles de Thaïlande présentent un haut degré de coopération et d'intégration. Bien qu'elles puissent être dans certains cas rivales, elles s'organisent malgré tout en un réseau global, défini autour d'un centre. On peut interpréter cette organisation par référence à la structure dialectale de la population hua ch'iao du pays. Pour des raisons historiques ${ }^{2}$, celle-ci est très largement dominée par les Teochiu de la région de Chaozhou, au nord de la province de Guangdong. En 1955, après la mise en place de quotas d'immigration très restrictifs, ils représentaient $56 \%$ de la population chinoise du pays, contre seulement $16 \%$ pour les Hakka, originaires du Fujian, le

2 - Taksin, le général qui restaura la grandeur du Siam après la cuisante défaite de 1767 face aux Birmans, était le fils d'un collecteur de taxes teochiu, qui avait été adopté par une famille aristocratique siamoise. Lui et ses successeurs sur le trône, qui avaient euxmêmes du sang chinois, donnèrent une impulsion décisive en faveur de l'immigration des membres de ce groupe dialectal. 
second groupe dialectal représenté sur place ${ }^{3}$. Cette hégémonie des Teochiu en Thaillande a d'autant plus favorisé l'intégration locale des shan tang que la préfecture de Shantou, d'où ils proviennent, semble avoir été un foyer très actif de ce genre d'institutions avant 1949. Il est significatif, à cet égard, que les plus influentes associations caritatives de Malaysia aient été créées par des hommes d'affaire teochiu, bien que, dans ce pays, les natifs de Shantou soient très minoritaires ${ }^{4}$.

Toutes les associations caritatives qui, en Thaillande, entrent dans le réseau évoqué plus haut ne s'auto-proclament pas shan tang. Seule une minorité d'entre elles revendiquent cette appellation dans leur intitulé officiel. Parmi elles, la plus ancienne et la plus importante par son niveau d'activités s'appelle Bao De Shan Tang (Po Têk Hsiang T'üng, «Maison de bienfaisance du retour de la vertu»). Trois autres groupes de temples-associations, organisés en lignées de culte et dont l'activité intègre les pratiques médiumniques de l'écriture révélée, admettent ce titre, bien qu'ils utilisent plus volontiers soit le terme de ge («pavillon », khor en teochiu), soit l'expression de shan tan (hsiang t'ua en teochiu), qui signifie "autel de bienfaisance », par allusion à l'autel médiumnique leur permettant d'entrer en communication avec leurs saints patrons. Ces trois lignées sont, par ordre d'introduction en Thaïlande: Ming T'ua, Pong Lai Hsiang T'ua et Chip T'ua. Enfin, la dernière lignée de culte introduite dans le pays est Têk K'a (De Jiao en mandarin). Comme les trois précédentes et à la différence de Po Têk Hsiang T'üng, son activité caritative est étroitement associée à la pratique de l'écriture révélée. Cependant, son médiumnisme revêt un caractère messianique, et elle se veut le fer de lance institutionnel d'un nouveau jiao, terme que les sinologues traduisent selon les cas par «doctrine» ou «corps de préceptes ». D'où le fait que les auteurs qui ont jusqu'à présent étudié le mouvement Têk K'a l'aient qualifié de secte, voire de religion ${ }^{5}$.

3 - Cf. Richard Coughlin, Double Identity. The Chinese in Modern Thailand, Hong Kong, Oxford University Press, 1960, p. 6. À titre de comparaison, la Malaysia présentait à la même époque un profil plus équilibré: les Hokkien du Fujian étaient 31,7\% des Hua Ch'iao; les Hakka et Cantonais, 21,8 et 21,7\% respectivement ; les Teochiu représentant pour leur part 12,1\% de l'effectif (VICTOR Purcell, The Chinese in Southeast Asia, Londres, Oxford University Press, 1965, p. 223).

4 - Selon le constat de Tan Chee-Beng ( "The Religion of the Chinese in Malaysia ", in Lee Kam Hing et Tan Chee-Beng (éds), The Chinese in Malaysia, Singapour, Oxford University Press, 1999, pp. 282-315), les temples du mouvement sectaire De Jiao en Malaysia furent pour la plupart créés par des hommes d'affaires teochiu. De plus, deux des principales shan tang du pays (l'une dans l'État de Perak et l'autre à Sarawak) sont organisées autour du bodhisattva teochiu Da Feng.

5 - Tan Chee-Beng parle de secte (The Development and Distribution of Dejiao Associations in Malaysia and Singapore: A Study on a Chinese Religious Organization, Singapour, Institute of Southeast Asian Studies, 1985), tandis que Kazuo Yoshihara préfère employer le concept de religion ( "Dejiao: A Chinese Religion in Southeast Asia », Journal of Religious Studies, 15-2, 1988, pp. 199-221). La traduction de jiao par "religion » étant contestée, le terme de secte me paraît mieux adapté, d'autant que De Jiao répond aux critères qui, en contexte chinois, justifient un tel usage selon David Jordan et DaNiEL Overmyer, The Flying Phonix: Aspects of Chinese Sectarianism in Taiwan, Princeton, Princeton Univer- 


\section{Po Têk Hsiang T'üng}

Cette Maison de bienfaisance, dont l'unique temple et les locaux administratifs sont situés au cœur du quartier chinois de Bangkok, est la plus ancienne et la plus puissante des associations caritatives de Thaïlande. La chronique de l'association attribue sa création au début du $\mathrm{Xx}^{\mathrm{e}}$ siècle à douze entrepreneurs teochiu, parmi lesquels trois jouèrent un rôle de premier plan. Le premier, Te Tiyong (Zheng Zhiyong en mandarin ${ }^{6}$ ), s'illustra dans les années 1890 en gérant la plus importante ferme des jeux du pays. Plus tard, il s'engagea dans la finance et plaça une partie de ses bénéfices dans l'achat de vastes terrains au cœur du quartier chinois. C'est à ce titre qu'il contribua à la création du temple, donnant la parcelle sur laquelle celui-ci est édifié. À sa mort, en 1935, un autel avec sa photographie lui fut consacré, et il devint le dieu du sol de ce lieu sous le titre de Te kong ("grand-père Te »). L'un des membres de son lignage, Huten Techaphaibun, à la tête d'un conglomérat de banques, dont la Bangkok Metropolis Bank, dirige Po Têk Hsiang T’üng depuis 1972.

Le second personnage est Tang Haosang. Né lui aussi au Siam, il fit fructifier dans la finance la fortune dont il hérita. Très riche, mais aussi humaniste, il apporta l'essentiel des fonds nécessaires à l'édification du temple dont l'architecture et l'aménagement se voulaient la réplique exacte du temple mère en Chine. La troisième figure est un négociant en fruits dénommé Be Himteng (Ma Runten en mandarin), qui avait immigré au Siam en 1880. En 1896, à la faveur d'un retour au pays natal, il transplanta au Siam le culte de Tai Hong (Da Feng en mandarin), dont il était un fervent dévot, rapportant avec lui lors de son équipée outre-mer de la poudre d'encens et surtout une statue en bois prise dans le plus ancien temple voué à ce saint bouddhiste en territoire teochiu, un temple qui s'appelait déjà Po Têk. Il installa un autel consacré à Tai Hong à son domicile, dont le culte était réservé aux membres de son lignage. Mais une série de miracles au bénéfice de ceux-ci et la réputation dont jouissait le saint dans la région de Shantou ${ }^{7}$ drainèrent vers la maison de Be Himteng un nombre croissant d'habitants du quartier chinois de Bangkok. Fort de ce succès, il créa une samakhom (association) en 1901, et, en 1910, après la construction du temple consacré à Tai Hong en plein cœur du quartier chinois sur le terrain donné par Te Tiyong, l'activité caritative du groupe fut reconnue d'utilité publique et la fondation Po Têk Hsiang T'üng fut officiellement instituée et placée sous patronage royal.

sity Press, 1986, p. 10, à savoir le rejet par le mouvement des institutions religieuses préexistantes.

6 - Hong Techawanich, de son nom thaï, était l'héritier d'une riche famille teochiu. Il naquit au Siam en 1851 et se rendit à Shantou pour un bref séjour d'études alors qu'il était adolescent.

7 - D'après Liu Wuchu, «Le culte de maître Da Feng en Thaïlande », Minjian Zongjiao (Popular Religion, 2, 1996, p. 6), à la fin du XIX ${ }^{\mathrm{e}}$ siècle, pas moins de cinquante-six shan tang organisaient leur activité charitable dans la région de Shantou autour du culte de Da Feng. 
Pour les Chinois de Thaïlande, Tai Hong symbolise au plus haut point la compassion bouddhique comme vertu efficace tout en faisant figure de héros culturel teochiu. C'est à ce double titre qu'il est vénéré dans la plupart des temples caritatifs chinois du pays. Il serait né en 1095, sous la dynastie des Song. Après avoir réussi ses examens mandarinaux, il fut nommé gouverneur d'un district, mais renonça rapidement à sa fonction pour se faire ordonner bonze au Fujian. Il pratiqua l'ascèse et la méditation dans les ruines d'un vieux monastère situé sur une colline du district de Chaoyang, au sud de Shantou. Grâce aux miracles qu'il réalisa par l'accumulation de ses mérites, il obtint les moyens de restaurer le monastère pour en faire un centre rayonnant du bouddhisme et, à partir de là, il chercha à améliorer le sort de ses contemporains. Lorsque la peste ravagea la région de Shantou au milieu du XII ${ }^{\mathrm{e}}$ siècle, il s'occupa des personnes touchées par l'épidémie et prit en charge l'inhumation des victimes qui n'avaient pas de parents. On lui prête aussi la construction du pont de la Paix, dans le district de Raoping, à l'embouchure du fleuve Liang, qui était redouté pour ses crues dévastatrices. Grâce à ses pouvoirs miraculeux, il aurait contenu l'écoulement de l'eau du fleuve durant sept jours pour permettre la réalisation d'une digue et du pont. Enfin, on devrait à Tai Hong l'invention de la fête du hsiu kou $k u^{8}$. La statue de bois de Tai Hong conservée par Po Têk Hsiang T'üng serait d'ailleurs l'effigie du saint portée en tête des processions qui, à Shantou, conduisaient périodiquement les restes des morts sans descendance sur le lieu de leur sépulture dans le cadre de cette fête.

Le principal objet du hsiu kou ku est d'offrir le salut à des morts sans descendance, qui, autrement, auraient erré sans fin dans la dimension invisible du monde des humains'. Ces défunts sont essentiellement des personnes d'ethnie thaïe qui, en vertu des croyances autochtones, sont des malemorts au destin tronqué. Dans ses modalités pratiques, le cycle rituel intègre le plus souvent une campagne de

8 - Certaines sources chinoises s'efforcent de faire la part de l'histoire et de la légende. C'est notamment le cas de l'article de Liu Wushu, « Le culte de maître Da Feng... », art. cit., p. 2. Selon cet auteur, Tai Hong/Da Feng aurait mis des années à collecter les fonds pour construire le pont de la Paix. Il supervisa les travaux de construction jusqu'à hauteur du seizième pilier, mais mourut avant de l'avoir achevé. Il revint aux villageois des environs de finir l'ouvrage. Le même auteur suggère que Da Feng n'aurait été qu'indirectement l'instigateur du rite du hsiu kou ku. De son vivant, il aurait certes insisté sur les mérites qu'entraînait la collecte des ossements des morts sans descendance, mais les Teochiu n'auraient pris conscience de l'efficacité de ces dispositions que plus tard, à l'époque des Ming et des Qing, lorsque de grandes calamités frappèrent la région à intervalles rapprochés. En diverses occasions, les dévots auraient alors promené l'effigie de Da Feng sur les lieux où étaient censés errer des malemorts afin de les rallier et de les diriger vers l'Au-delà. Ces actions se seraient avérées efficaces pour éloigner l'infortune, d'après l'interprétation des locaux. De tels prodiges accrurent la popularité de Da Feng et de ses commandements, la liturgie de la fête prenant sans doute progressivement forme à partir de ces prémisses.

9 - Voir Bernard Formoso, «Hsiu-kou-ku, The Ritual Refining of the Restless Ghosts Among the Chinese of Thailand ", Journal of the Royal Anthropological Institute, 2-2, 1996, pp. 217-234. 
quête médiumnique de restes humains dans les zones de forêt où les Thaïs inhument leurs mauvais morts : suicidés, accidentés, victimes d'homicides ou de maladies conduisant à une fin prématurée. Cette quête est plus ou moins remplacée par celle des cadavres d'accidentés de la circulation et autres morts que les shan tang collectent au fil de leurs interventions humanitaires et qu'elles stockent dans leurs cimetières. Les restes sont ensuite purifiés au travers de divers rites, puis expédiés dans l'Au-delà par l'entremise d'une crémation collective.

\section{Ming et Chip T'ua}

Ces deux lignées de culte sont indissociables, puisque la seconde est une branche dissidente de la première. Ming T'ua («autel de la clarté ») est apparue dans le pays en 1925. Son premier temple fut construit dans la province de Chonburi, plus précisément dans la ville de Siracha, à cent kilomètres au sud-est de Bangkok. L'encens qui permit sa fondation fut prélevé par un groupe d'immigrés teochiu dans un temple voué aux Huit Immortels (Poi Hsian(g) en teochiu) de la ville de Puning, au sud de Shantou. Au sein du groupe des immigrants, un homme avait jadis servi de médium, assurant jusqu'à sa mort cette fonction dans la localité de Siracha. À partir de ce point d'ancrage, le culte des Huit Immortels se popularisa et essaima parmi les Teochiu de Chonburi. Entre 1926 et 1940, six temples Ming furent créés dans cette province (cf. cartes p. 842). Puis, après la Seconde Guerre mondiale, la lignée de culte essaima dans les différentes régions du pays, avec cependant une plus forte implantation dans le Centre et le Nord-Est. En l'an 2000, on compte quarante-quatre temples affiliés au mouvement caritatif Ming T'ua en Thaïlande ${ }^{10}$.

Le nombre de temples Ming aurait pu être plus important s'il ne s'était produit en 1969 un incident qui poussa un temple à la dissidence. Ses adeptes fondèrent alors un nouveau mouvement, appelé Chip T'ua ( unité - autel»), qui est resté malgré tout embryonnaire, puisqu'il ne compte en l'an 2000 que deux établissements implantés dans le nord-est du pays. La lignée s'organise comme Ming T'ua autour du culte des Huit Immortels et il n'y a aucune différence sur le plan liturgique entre les deux mouvements. Leur différend est né d'une question de préséance liée au prêt d'une statue.

\section{Pong Lai}

Cette lignée de temples, dénommée par référence aux îles de Peng Lai (province de Shandong) qui sont le lieu de résidence mythique d'Immortels du panthéon taoïste, s'est développée en Thaïlande dans les années 1950. L’histoire locale du culte à Ho Hiahun, le patron de la lignée, date de la fin du XVIII ${ }^{\mathrm{e}}$ siècle. Elle est si indicative du processus de transposition au Siam de multiples pratiques 
dévotionnelles chinoises qu'elle mérite d'être brièvement retracée. Elle remonte au début du XIX $X^{\mathrm{e}}$ siècle, époque où les premiers rois Chakri incitèrent les Teochiu à immigrer au Siam pour faciliter son développement. Dans le cadre de cette politique, les habitants d'un marché de Puning, un district proche de Shantou, affluèrent en nombre pour s'établir dans la localité de Phan Thong, province de Chonburi. L'immigration fut si massive que le nouveau marché reprit le nom de celui de Puning - Pun Thao Ma - et que les trois divinités qui protégeaient l'ancien établissement firent le voyage, à savoir: Guan Yin (Avalokitesvara, dans sa version chinoise), le dieu de la cité et Ho Hiahun. Ce dernier, parce qu'il était le moins important des trois, fut longtemps négligé. Il en prit ombrage d'où, expliquent les adeptes de son culte, une forte récession économique qui toucha Phan Thong au sortir de la Seconde Guerre mondiale. Deux Teochiu, qui étaient versés dans l'écriture révélée et se souvenaient du chant d'invitation de la divinité, s'efforcèrent d'entrer en communication avec lui en 1951. Au cours des premières séances médiumniques, Ho Hiahun ne se manifesta que par l'entremise d'une déité tierce pour afficher son mécontentement, puis demanda que le déficit dévotionnel dont il avait été victime fût compensé par cinq journées ininterrompues d'opéra chinois organisées en son honneur. Cette volonté fut respectée. Le dieu s'exprima alors directement et demanda que fût créée une association du nom de Pong Lai à Phan Thong, à l'est de Bangkok. Progressivement, de nouveaux lieux de culte se constituèrent par partage de l'encens à partir de ce temple mère. On en dénombrait vingt-huit en l'an 2000 (voir cartes p. 842).

Ho Hiahun (He Yeyun en mandarin) serait né en 776 de notre ère dans la province de Guangdong. Il fut d'abord devin et géomancien à la cour impériale, mais il tomba en disgrâce lors d'une lutte de succession au sein de la dynastie des Tang. Il changea alors de nom pour se faire appeler Ho Hiahun, adopta un mode de vie ascétique et erra en pays hokkien (Fujian) en quête d'un ermitage forestier qui lui permît de s'adonner paisiblement à la méditation. Mobilisant toute son énergie pour accroître ses pouvoirs de géomancien, il négligea son apparence physique, au point qu'on le baptisa par dérision : "Immortel plein de puces » (Sak $\mathrm{B}$ Hsiang en teochiu). Il sillonna pendant cinq ans le pays teochiu et se rendit très populaire par ses divinations. À la fin de sa vie, il entreprit d'enseigner la voie ascétique du bouddhisme. Après sa mort, il fut vénéré non seulement comme fameux géomancien et devin, mais aussi comme Immortel incorporant le groupe de Peng Lai, d'où le nom de l'association. De plus, il aurait atteint le nirvāna selon ses adeptes. Sa tombe, située dans le district teochiu de Tiahia, est devenue un important lieu de pèlerinage.

Les figures de Tai Hong et Ho Hiahun sont complémentaires. En effet, si le premier est étroitement identifié au pays teochiu, Ho Hiahun fit toute sa vie le lien entre les zones de peuplement teochiu et hokkien. Par ailleurs, il existe un large consensus parmi les Chinois de Thaïlande pour reconnaître à Tai Hong le statut de phothisat (bodhisattva en thaii). Il fait figure de saint bouddhiste engagé sur la voie de la perfection mais qui diffère son illumination pour prêcher la doctrine et aider son prochain. Or, cette orientation mondaine le distingue de l'anachorète Ho Hiahun qui se retira du monde pour renforcer ses pouvoirs et finalement 
atteindre le nirvāna. Autant donc le premier s'illustre dans le «bouddhisme social » en incarnant au plus haut point l'idéal de compassion, autant le second sert d'icône à la voie ascétique. Ajoutons enfin que Ho Hiahun est une figure polyvalente. Son hagiographie le présente tour à tour comme le patron des géomanciens et des astrologues, comme un «Immortel» taoïste et comme un Bouddha.

\section{Têk K'a (De Jiao)}

C'est en 1939 que Têk K'a («enseignement de la vertu», De Jiao en mandarin) émergea comme mouvement religieux dans la région de Shantou, à l'initiative de trois Teochiu. Ceux-ci, au terme d'un mois de jeûne, avaient reçu lors d'une séance médiumnique dans un temple du district de Chaoyang un message émanant de deux divinités taoïstes, Liu Ch'unwong et Ya Hungsang. Elles leur ordonnaient de fonder un nouveau courant religieux pour sauver le monde et les incitaient à fournir aux indigents une assistance matérielle et sanitaire. Durant la Seconde Guerre mondiale, le mouvement prit de l'ampleur au point d'intégrer vingt-cinq associations et de compter comme adeptes près de dix mille maisonnées. Toutes les associations furent désignées par l'idéogramme générique Chi ("pourpre»), qui demeure aujourd'hui encore l'emblème de la secte.

La Thaïlande fut, après la Chine et Hong Kong, le troisième pays où le mouvement se propagea, à l'initiative d'un homme d'affaire nommé Lin Hsiuwu. Celui-ci, né au Siam, avait été envoyé dans les années 1910 à Shantou pour son éducation. Bloqué en Chine du fait de la guerre sino-japonaise, il devint adepte de Têk K'a et fréquenta avec assiduité l'un de ses temples, Chi Hsiong khor, situé à Shantou. En 1945, alors qu'il s'apprêtait à rentrer en Thaïlande, Ch'iah Hsiant'ong, un maître taoïste qui participait du groupe des divinités tutélaires du temple, le choisit pour propager la foi Têk K'a en Asie du Sud-Est. Lors de la séance médiumnique où il édicta ce commandement, Ch'iah Hsiant'ong traça par l'entremise d'un médium âgé de treize ans seulement les portraits des figures du panthéon Têk K'a afin que Lin Hsiuwu les emportât avec lui. Puis, comme ce dernier redoutait la tâche qu'on lui confiait, il le rassura en lui indiquant qu'il s'occuperait de tout et qu'il se manifesterait à lui, au besoin, sous les traits d'un grand goéland. En 1949, inspiré par un rêve, Lin Hsiuwu contacta le médium principal du temple de Shantou, un dénommé Hia Senglu, et l'aida à immigrer en Thaïlande avec quelques-uns de ses collègues. Ensemble ils organisèrent des séances médiumniques au domicile de Lin. Leur audience s'accrut alors très vite et, par le partage de l'encens, ils ouvrirent dans les mois qui suivirent un autre lieu de culte à Bangkok. Un riche homme d'affaires nommé Li Chihmian prit alors la tête du mouvement. Il était à la fois président de la puissante Association des exportateurs de riz et vice-président de la chambre de commerce de Bangkok. Sa renommée draina vers Têk K'a des personnalités de premier plan, dont la bellemère du maréchal Phibun Songkhram, alors dirigeant du pays, qui facilita son enregistrement comme association caritative en 1953, malgré l'hostilité déclarée du gouvernement thaïlandais de l'époque à l'encontre de la minorité chinoise. 
À partir des années 1960-1970, Têk K'a essaima d'autant plus rapidement dans le pays que le contexte économique et politique devint favorable et qu'elle posait bien moins de conditions à l'ouverture de nouveaux temples que ses concurrents Pong Lai ou Ming T'ua. En 1960 elle comptait déjà huit composantes dans le pays, chiffre qui passa à 33 en 1980 et à 58 en 2000 (cf. cartes p. 842), la plaçant ainsi en tête des organisations caritatives de Thaïlande par le nombre de ses agences. À partir de Shantou, le mouvement s'implanta aussi en Malaysia, à Singapour et dans d'autres pays de la région, à Hong Kong, au Laos, à Taïwan, aux États-Unis et, plus récemment, en Chine populaire elle-même. Ces différentes branches nationales se sont regroupées depuis 1987 en une fédération, la Nangyang Moral Uplifting (De Jiao) General Society, qui organise, tous les trois ans, un World Moral Divine Meeting au cours duquel les saints patrons de la fédération délivrent, par le biais de médiums, des oracles ou des préceptes en teochiu, mandarin, thaï, malais et même, plus récemment, en anglais.

D'un pays à l'autre les déités qui font l'objet d'un culte dans les temples Têk K'a sont variables. Kazuo Yoshihara et Tan Chee-Beng observent que les branches de Hong Kong, de Singapour et de Malaysia se veulent syncrétiques, puisque, agissant sur un mode universaliste, elles mettent l'accent sur les wu jiao, les «cinq doctrines». Elles ont en effet intégré dans leur panthéon les figures emblématiques de Lao Tseu, de Confucius, de Bouddha, de Jésus et de Mahomet $^{11}$. Or, dans un pays comme la Thaïlande où le bouddhisme est religion d'État, un tel élargissement n'est pas de mise. Parmi les cinq piliers du culte local que sont Liu Ch'unwong, Ya Hungsang Wu Manghu, Ch'iah Hsiant'ong (quatre figures du taoïsme populaire) et Chi Kong (un saint du bouddhisme), c'est ce dernier qui prévaut, au côté du bodhisattva teochiu Tai Hong.

La légende de Chi Kong est bien connue. Il aurait vécu au XII siècle de notre ère à Hangzhou, qui était alors la capitale des Song du Sud. Orphelin et ordonné bonze jeune, il s'illustra rapidement dans l'apprentissage de la doctrine bouddhique. Néanmoins, ses préceptes étaient plus exemplaires que sa pratique, et il devint fameux pour ses beuveries et ses excentricités. De telles frasques, mais aussi une capacité exceptionnelle à guérir les malades, à repousser les forces démoniaques ou à résoudre les litiges le firent passer à la postérité sous le titre de "guérisseur fou». Certaines des maximes qui lui sont attribuées inspirent l'action philanthropique de Têk K'a, ainsi que les «dix vertus» dont le mouvement se veut le promoteur (piété filiale, loyauté, obéissance, rectitude morale, bienséance, foi, sagesse, générosité, souci de la face et bienveillance). 
Pong Lai
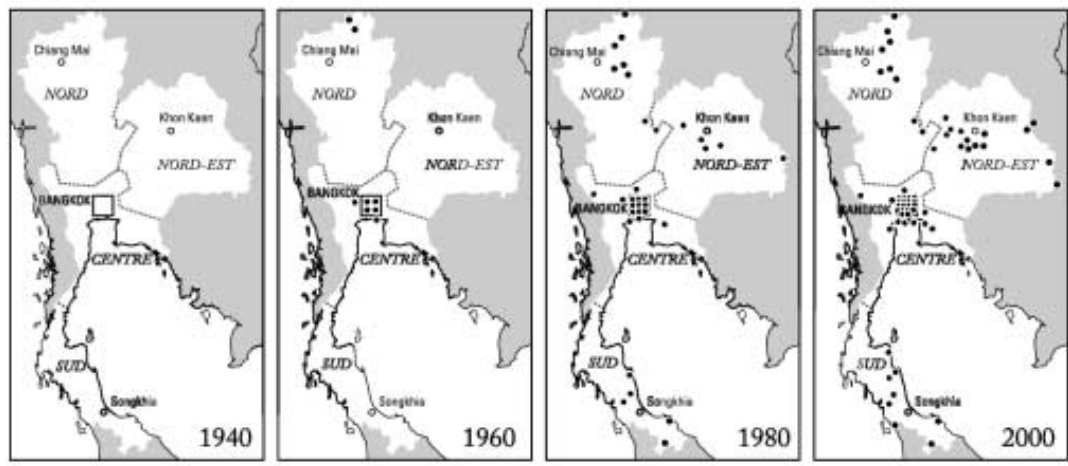

Ming et Chip T'ua
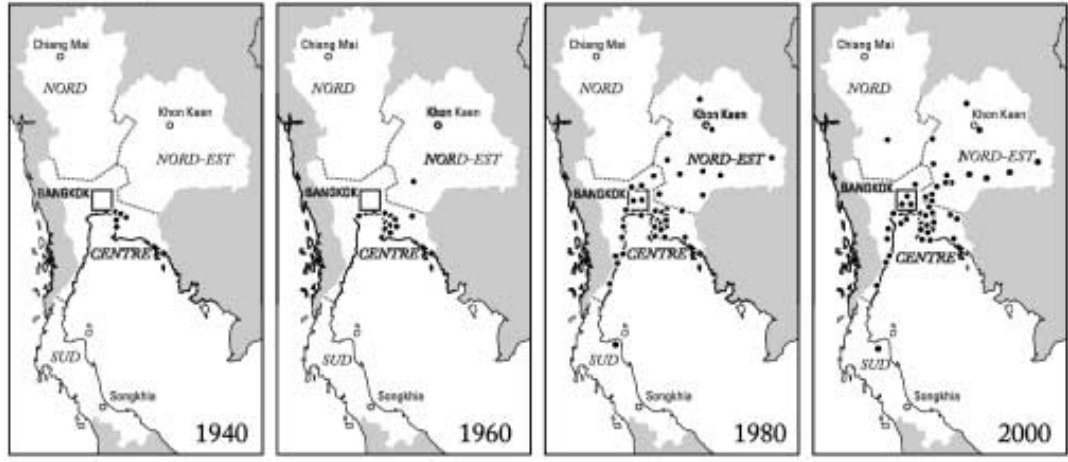

Têk K'a (De Jiao)
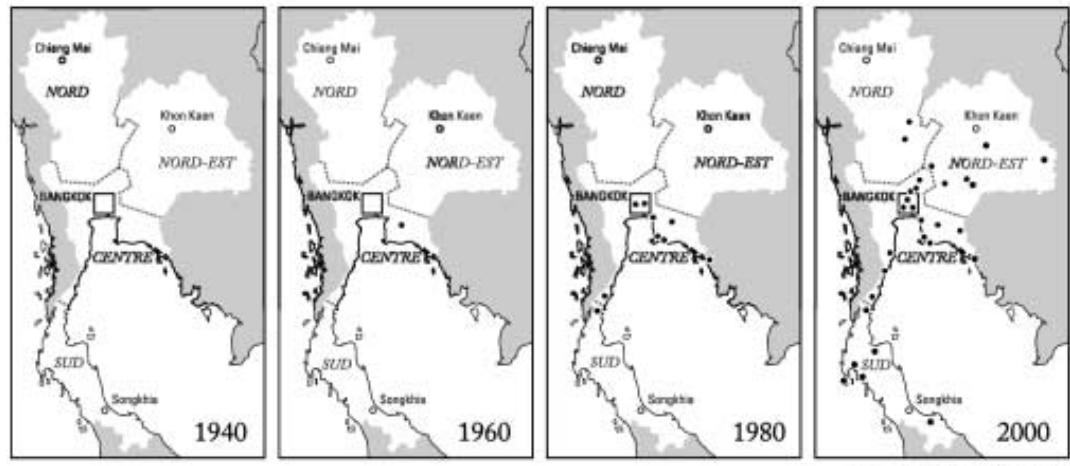

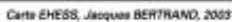




\section{Quatre lignées autour d'un centre}

Dans la numérologie chinoise, le chiffre cinq est le symbole du centre comme quintessence du tout, et c'est sur son mode que se déclinent les lois universelles, qu'elles aient trait aux couleurs, aux saveurs, aux sons, aux métaux, aux viscères, aux planètes, etc. Or, de manière semble-t-il fortuite, les associations chinoises à vocation caritative de Thaïlande se répartissent depuis la fin des années 1960 en quatre lignées gravitant autour d'une shan tang au statut particulier : Po Têk Hsiang T'üng.

Les facteurs de leur unité en un réseau global sont multiples. Le plus fondamental est l'identité teochiu de la plupart des fondateurs et animateurs de ces institutions. À l'échelle de chaque ville, les uns et les autres forment un petit noyau de gens volontaires, soucieux de préserver les traditions de leur pays natal ou de leur terre ancestrale. Aussi coopèrent-ils dans le cadre d'autres associations servant ces objectifs : comité du temple aux dieux du territoire, comité de l'école chinoise de la ville ou association des natifs de tel ou tel district teochiu. Qu'ils soient sympathisants de Ming T'ua, de Têk K'a ou de Pong Lai influe peu sur la teneur généralement cordiale de leurs rapports.

Concernant le maintien d'une identité régionale propre aux natifs de Shantou, Ming T'ua, Chip T'ua, Pong Lai et Têk K'a vouent un culte au bodhisattva teochiu Tai Hong. Certes, à la différence des déités propres à chacune de ces lignées, Tai Hong ne communique généralement pas avec les dévots via l'écriture inspirée des médiums ${ }^{12}$. Néanmoins, il sert d'icône légendaire à l'abnégation et à la compassion des gens originaires de cette partie septentrionale du Guangdong. Le culte qu'on lui voue trouve aussi sa raison d'être dans la fête décennale du hsiu kou ku dont il aurait été, selon la légende, l'inventeur.

La fête mobilise des moyens financiers et humains importants. Aussi le nombre de ses occurrences et le faste de ses célébrations servent-ils d'étalon de renommée en même temps que de comput historique aux associations caritatives hua ch'iao du pays. Po Têk Hsiang T'üng doit ainsi une part de sa prééminence institutionnelle au fait qu'elle soit la seule Maison de bienfaisance à avoir organisé dix hsiu kou ku qui, de surcroît, lui ont permis de recycler 153000 cadavres, selon le décompte de $1999^{13}$. À titre de comparaison, Ming Teng, le plus ancien temple

12 - Le seul contre-exemple que nous connaissions est celui de la plus grande shan tang du sud de la Thaïlande, Thong Sia hsiang T'üng de Hat Yai, où, lors des grandes fêtes (hsiu kou ku ou fête du Salut universel), il est fait appel à des médiums de l'association Têk K'a de la ville pour communiquer avec Tai Hong.

13 - Ces hsiu kou ku durèrent de trois à sept jours. Le premier, organisé en 1936, aboutit à l'incinération de 1028 cadavres; le deuxième, en 1947, à celle de 4095 ; le troisième, en 1955, à celle de 13044 personnes, le quatrième, en 1960 à 16023 cadavres; le cinquième, en 1965, à 17240 ; le sixième, en 1971, à 19241 ; le septième, en 1980, à 30 584; le huitième, à l'incinération de 13 459; le neuvième en 1994 à 18206 corps et, enfin, le dixième, à 20347 cadavres recyclés (je dois ces informations aux rapports d'activité de l'association publiés en 1990 et 1993, auxquels s'ajoutent les données livrées par son actuel dirigeant, M. Chayaphong Wisemongkhonchai). 
Ming de Thaïlande situé à Siracha, n'en était qu'à sa neuvième fête en 2000 et n'avait traité, de l'aveu même de ses responsables, que cinquante mille corps, soit trois fois moins.

Bien que l'initiative d'organiser un hsiu kou ku soit locale, chacun de ces événements revêt une dimension nationale. Il est d'usage en effet que le temple organisateur envoie des cartons d'invitation et le programme de la fête à toutes les agences des quatre autres organisations caritatives. Par ce biais, il s'agit de faire une large publicité à l'événement, mais aussi de rallier des équipes de volontaires qui aideront à déterrer les cadavres des malemorts pour les expédier dans l'Audelà et qui, en retour, se verront non seulement offrir le gîte et le couvert, mais accroitront aussi leur lot de mérites et leur bonne fortune. L'observation ethnographique révèle que se produisent en ces circonstances des chassés-croisés importants de main-d'œuvre entre les lignées de culte et les communautés hua ch'iao des différentes régions de Thaïlande. C'est l'occasion pour ces dévots d'échanger des informations, des expériences, des points de vue et, d'une certaine manière, de donner corps à la communauté imaginée des Chinois du pays. Une autre circonstance où se réalisent ces coalescences éphémères tient aux désastres d'envergure nationale vers lesquels convergent les équipes de sauveteurs des différentes shan tang ${ }^{14}$.

Les échanges de services religieux auxquels se livrent les diverses lignées de temples sont un autre facteur d'unité qui dénote aussi leur non-prosélytisme. En effet, les équipes de prêtres chinois étant rares dans les provinces thaïlandaises, il est courant que les temples d'une lignée aient recours au service d'officiants des temples d'une autre, située dans leur voisinage. C'est en particulier le cas de Têk $\mathrm{K}$ 'a, dont la croissance rapide ces dernières années (trente agences créées entre 1980 et 2000) n'a pu s'accompagner de la formation de prêtres et de médiums qualifiés en nombre suffisant ${ }^{15}$. Du coup, on assiste à l'apparent paradoxe d'un mouvement sectaire qui se veut nouveau «corps de préceptes" ou "nouvelle religion », et qui, pourtant, en certains de ses temples, dépend de la bonne volonté d'un personnel extérieur pour la conduite des rites élémentaires. En 2000, par exemple, l'agence Têk K'a de la ville de Chumpae invita les prêtres de l'association Chip T'ua de la cité voisine de Khon Kaen à réciter les sūtra bouddhiques nécessaires à la conduite de la fête du Salut universel. Ce paradoxe se résout si l'on considère que le syncrétisme de Têk K'a tient plus à sa volonté de fédérer sous son emblème les messages d'amour universel délivrés par les grands courants religieux (taoïsme, bouddhisme, islam, christianisme) qu'à produire un dogme spécifique et une liturgie adaptée.

14 - Ainsi, lors de l'effondrement de l'hôtel Royal Plazza de Khorat en 1993, près de trois cents sauveteurs appartenant non seulement à l'association Ming T'ua de la ville, mais aussi à Po Têk Hsiang T'üng et à diverses agences Têk K'a du Nord-Est travaillèrent de concert pendant plusieurs jours pour exhumer des décombres les quelques survivants du sinistre.

15 - Ainsi, en 2000, Têk K'a ne disposait à l'échelle de l'ensemble du pays que d'une dizaine d'équipes de médiums pour près de soixante agences, tandis que presque toutes les associations Ming T’ua et Pong Lai possédaient au moins une équipe. 
Les shan tang ou assimilées se rejoignent aussi sur le plan de l'action humanitaire en appliquant aux mêmes fins des moyens de nature équivalente. À Bangkok, par exemple, Po Têk Hsiang T'üng et certaines associations Têk K'a rivalisent depuis des décennies sur certains secteurs d'activités comme l'assistance médicale apportée aux indigents ou la prise en charge des morts. Po Têk Hsiang T'üng gère ainsi depuis 1973 un hôpital d'une capacité d'accueil de sept cent cinquante lits, situé en plein cœur du quartier chinois, tandis que l'une des premières associations Têk K'a à avoir été créée, Chi Hiang Khor, administrait jusqu'à il y a peu un autre hôpital de cinquante places, localisé lui aussi au cœur de la capitale. Quant à l'une de ses consœurs, Chi Mui Khor, ouverte au début des années 1960, elle offre des soins gratuits aux pauvres via trois dispensaires localisés dans Bangkok ou sa banlieue. De plus, Chi Hiang Khor possède le plus grand cimetière chinois de Thaïlande et a, pendant plusieurs décennies, recueilli autant de corps abandonnés que Po Têk Hsiang T'üng, ceci par le biais de réseaux d'approvisionnement d'ampleur comparable.

Néanmoins, la solidarité vocationnelle et ethnique de ces associations l'emporte sur la concurrence issue de leur proximité géographique ou du redoublement de leurs activités. Ainsi, pour prolonger l'exemple précédent, Chi Hiang Khor et Chi Mui Khor participent avec Po Têk Hsiang T'üng d'une même organisation: les «Cinq grandes maisons de bienfaisance » de Bangkok (ngow ta hsiang t'ïng en teochiu). Les membres de ce pool se réunissent chaque année à la veille du Nouvel An chinois et du Nouvel An thaï pour coordonner les offrandes alimentaires et monétaires qu'ils font en ces deux occasions aux hospices et prisons de la capitale. Ils se retrouvent aussi plus épisodiquement pour se répartir les interventions d'urgence en cas de désastres affectant Bangkok et ses environs.

Pour clore ces remarques sur les facteurs qui sous-tendent l'unité des shan tang de Thaïlande et des associations assimilées, il faut aussi évoquer ce principe de hiérarchisation commun qui veut que le temple d'où l'encens fut prélevé pour donner vie à un autre lieu de culte conserve toujours un ascendant symbolique sur ce dernier ${ }^{16}$. Aucune agence locale ne peut ainsi organiser de hsiu kou ku sans en

16 - Concernant les logiques d'extension et de hiérarchisation des lignées de culte, notons que Pong Lai et Têk K'a procèdent à un partage de l'encens mono-centré, puisque chaque nouvelle agence prélève de la poudre auprès du premier temple édifié dans le pays, tandis que Ming T'ua se développe selon un mode qui mêle l'ordre chronologique au critère de la proximité. Le temple dans lequel l'encens est prélevé est le plus récent de la lignée, dès lors qu'il n'est pas trop distant. Ainsi, l'agence Ming T'ua de Khorat, la plus ancienne du Nord-Est, a donné naissance à celle de Udon Thani, qui est à son tour à l'origine du temple Ming d'Ubon Ratchatani, dont dérive celui de Buriram. Entre-temps, des temples du mouvement avaient aussi été créés dans la Plaine centrale, mais le strict ordonnancement chronologique céda le pas au critère de la proximité (Udon, Ubon et Buriram étant des villes du Nord-Est, comme Khorat). Pour être complet, ajoutons que la hiérarchie juridictionnelle importe également. En effet, les temples des villes d'une même province tiennent leur encens de celui de l'agence du chef-lieu dès lors qu'elle existe. 
avoir auparavant référé à son temple mère et lui avoir emprunté, le temps de la fête, le brûle-parfum identifié aux saints patrons du mouvement.

Dans l'organisation de toutes les shan tang et associations assimilées du pays en une commune structure, le temple de Po Têk Hsiang T'üng est le centre. La prééminence de cette association tient au fait qu'elle est la plus ancienne Maison de bienfaisance du pays, qu'elle ait introduit au Siam le culte de Tai Hong dans lequel se reconnaissent toutes les autres lignées, qu'elle ait organisé le plus grand nombre de hsiu kou ku, mais aussi qu'elle soit financièrement la plus puissante. Elle concentre, en effet, des richesses d'une ampleur exceptionnelle dont elle redistribue une part aux quatre autres lignées d'associations caritatives qui l'entourent. Fondée, puis dirigée par certains des plus grands banquiers du pays, Po Têk Hsiang T'üng fait ainsi figure de «banque » du mouvement associatif chinois. Entre 1983 et 1993, elle a collecté et redistribué sous diverses formes 28,1 millions de dollars nord-américains et, en 1993, son patrimoine était estimé à 110 millions de dollars (à titre de comparaison, Têk K'a n'en a recueilli durant la même période que 19,5 millions). Rien que pour la construction, achevée en 1992, d'un centre universitaire de pointe pour la formation des personnels de santé et qui comptait en 2003 près de trois mille étudiants, l'association a collecté près de vingt millions de dollars. L'association est riche parce que les magnats de la finance qui la dirigent sont généreux et qu'ils font jouer leurs réseaux pour drainer vers le temple d'importantes donations. D'autre part, du fait de son ancienneté, Po Têk Hsiang T'üng est bien connue dans le pays et ses œuvres jouissent d'une grande publicité dans les médias. Enfin, le trait de génie de ses dirigeants est d'avoir fait de l'association le pivot d'un système de collecte de fonds et de subventions qui bénéficient à l'ensemble du mouvement associatif chinois.

Comme dans tout système redistributif, le mouvement est d'abord orienté vers le centre : une fois par an, à l'approche de la fête du Printemps, des correspondants locaux ou des émissaires en provenance de Bangkok écument les bourgades et villes du pays afin de recueillir des dons auprès du plus grand nombre possible de foyers chinois ou sino-thaïs. En échange de leur contribution, ces foyers reçoivent des talismans placés sous le sceau de Tai Hong pour protéger leur maison contre les forces maléfiques. Cette campagne de collecte de fonds est conduite grâce au soutien logistique que fournissent les agences locales de Têk K'a, de Ming T'ua, de Chip T'ua ou de Pong Lai. Les responsables de ces agences se doivent de faciliter la tâche du délégué de Po Têk Hsiang'T'üng en lui prêtant un véhicule, en l'introduisant dans les cercles locaux influents ou bien en lui fournissant des accompagnateurs. Le même mouvement centripète opère au niveau des associations chinoises de la capitale. Po Têk Hsiang T'üng entretenait en l'an 2000 des relations de partenariat avec toutes les ch'iao t'uan, ou associations par lieu d'origine basées à Bangkok, ainsi qu'avec sept journaux d'expression chinoise, la Fédération nationale des associations claniques, la chambre de commerce sinothaïlandaise, diverses organisations fonctionnelles (Association de promotion de la culture chinoise, fondations animées par des médecins, hôpital chinois, etc.), ou bien encore avec plusieurs sociétés bouddhiques. Qui plus est, elle avait tissé des 
relations privilégiées, concrétisées par plusieurs réunions annuelles, avec quatre des cinq autres shan tang de la capitale, parmi lesquelles deux appartiennent au mouvement Têk K'a. Ensemble, elles forment les ngow ta hsiang t'üng, les «cinq grandes maisons de bienfaisance », comme indiqué précédemment. En tout, c'est près de deux cents associations hua ch'iao parmi les plus importantes du pays qui forment un « réseau d'organisations philanthropiques parentes ou amies » (hongkon khrüakhai phan mit banthaophai). Elles collectent des dons une fois par an auprès de leurs adhérents pour le compte de Po Têk Hsiang T'üng.

En retour, celle-ci finance sur dossier une partie de leurs projets humanitaires (doter un village pauvre d'un réseau d'eau courante ou de fournitures scolaires, par exemple, ou bien renouveler l'équipement de liaison radio de leurs véhicules d'intervention). Elle utilise aussi parfois les organisations alliées comme relais de sa propre action caritative. C'est le cas depuis 1997 pour la distribution de lots de denrées de base aux nécessiteux qui clôt la fête du Salut universel. Comme cette distribution de masse paralysait le trafic automobile et perturbait l'ordre public au sein du quartier chinois, les responsables de l'association ont décidé depuis cette date de n'en allouer qu'un contingent symbolique à partir du temple (de l'ordre de cinq mille lots), les autres l'étant par le réseau des organisations "parentes ou amies » de la capitale. Enfin, dans chacune des quatre régions du pays (le Centre, le Sud, le Nord et le Nord-Est), les représentants locaux de Pong Lai, Têk K'a, Ming T'ua et Chip T'ua se retrouvent au sein d'une commission qui se réunit en cas de catastrophe. Cette commission, où siègent aussi des représentants de l'État (gouverneurs de province, chefs de la police, directeurs d'hôpitaux), coordonne l'intervention des secours et lève le plus rapidement possible des fonds pour porter assistance aux victimes. Or, il est d'usage que Po Têk Hsiang T'üng contribue à hauteur de $10 \%$ des sommes globales engagées dans ces opérations.

Finalement, les rapports que Po Têk Hsiang 'T'üng entretient avec les autres Maisons de bienfaisance et, plus largement, avec les différentes associations chinoises du pays peuvent être interprétés sur le mode du patronage. Parcourir une fois l'an les provinces du pays est certes pour ses émissaires un moyen de collecter des fonds et de placer le plus grand nombre possible de Hua Ch'iao sous la protection occulte de Tai Hong, mais c'est aussi l'occasion de vérifier l'allégeance des autres associations caritatives, dont les agences locales, à la manière de fidèles vassaux, doivent se placer au service de ses intérêts supérieurs. En retour, à grands renforts de subventions octroyées par concours, l'association entretient sa clientèle et délivre des brevets d'action humanitaire. Cette structure hiérarchique est d'autant plus remarquable qu'elle n'a en rien été affectée par l'expansion rapide du réseau caritatif. En effet, bien que le nombre de Maisons ou Autels de bienfaisance ait augmenté de manière exponentielle depuis un siècle, avec au moins un doublement tous les vingt ans ( 7 temples en 1940, 22 en 1960, 64 en 1980 et 133 en 2000), la structure mono-centrée que nous avons décrite reste pleinement opératoire.

Les modalités d'une telle expansion prêtent à commentaires tant elles reflètent l'évolution politique et socio-économique de la communauté hua ch'iao du pays, ainsi que sa répartition sur le territoire national. Si plus de $70 \%$ des temples 
caritatifs chinois que compte le pays en 2000 ont été créés après 1960, c'est pour deux raisons essentielles. D'abord, parce que l'ostracisme à l'encontre des Chinois qui avait marqué entre 1938 et 1958 la politique des gouvernements nationalistes siamois se relâcha au début des années 1960 ; ensuite, parce qu'à partir de cette date un enchaînement de facteurs favorables (l'aide américaine, l'essor corrélatif de l'agriculture commerciale d'exportation, puis les progrès spectaculaires de l'industrie) assurèrent au pays une croissance économique forte, de l'ordre de $8 \%$ par an. Or, du fait de leur position dominante dans les secteurs du commerce, de la finance et de l'industrie, les Chinois comme les éléments métissés sino-thaïs profitèrent plus que toutes les autres composantes de la nation de cette croissance. Le développement de l'action philanthropique chinoise à compter de cette période, et surtout entre 1984 et 1997 (trente-six temples construits en treize ans), qui correspond au boom économique thaïlandais, fut la conséquence directe de la prospérité atteinte par la majorité des Hua Ch'iao et de l'enrichissement spectaculaire d'une petite partie d'entre eux.

La distribution spatiale des temples témoigne pour sa part assez fidèlement de la répartition inégale des Hua Ch’iao dans le pays. En 1947, 65 \% des Chinois se concentraient dans le bas Siam et la région du golfe, les régions périphériques du Nord, du Nord-Est et du Sud ne regroupant chacune que $11 \%$ des immigrés ${ }^{17}$. Depuis lors, la situation n'a guère évolué du fait de l'hyper-concentration de l'activité industrielle et financière du pays à Bangkok et dans la Plaine centrale ${ }^{18}$. De plus, les Hua Ch'iao sont largement urbains (selon certaines estimations plus de $50 \%$ des citadins de Thaïlande ont du sang chinois) et, là encore, la Plaine centrale tranche par rapport au reste du pays avec un taux d'urbanisation de $38 \%$ contre 8,5 à 5,5\% pour les autres régions, Bangkok étant par ailleurs en 1993 d'une taille quarante fois supérieure à la seconde ville du pays ${ }^{19}$. En croisant ces données : taux d'urbanisation par région, proportion de Thaïlandais d'ascendance chinoise dans les villes et tendances récentes en matière de déconcentration entrepreneuriale, on aboutit aux chiffres suivants en matière de répartition géographique des Hua Ch'iao dans le pays: $70 \%$ à Bangkok et dans la Plaine centrale en 1993, contre $15 \%$ dans le Nord-Est, 7,5\% dans le Nord et dans le Sud. Or, la répartition des shan tang dans le pays reproduit à peu près ces masses: lorsque l'on additionne le nombre des temples de Bangkok et ceux de la Plaine centrale, on atteint ainsi la proportion de $54,5 \%$ (72 établissements), pour $25,5 \%$ dans le Nord-Est (34 temples), $9 \%$ dans le Nord (12 shan tang) et $11 \%$ (15 temples) dans le Sud.

17 - Selon les données fournies par William T. Skinner, A Study of Chinese Community Leadership in Bangkok, Together with an Historical Survey of Chinese Society in Thailand, Ph. D., Cornell University, 1954, p. 246.

18 - Au début des années 1990, la capitale et sa périphérie concentraient plus des deux tiers de l'activité industrielle du pays, près de la moitié du PIB, environ $60 \%$ des services, un pourcentage identique des structures hôtelières et captaient presque $70 \%$ de l'épargne nationale.

19 - Cf. 1993. Thailand Population Data Sheet, Bangkok, Chulalongkorn University/Institute of Population Studies. 
Pour clore ces propos sur le déploiement des temples caritatifs chinois dans le pays, on peut s'interroger sur les motivations qui poussent un noyau d'entrepreneurs à engager des moyens financiers aussi considérables afin d'instituer dans leur ville une shan tang. L'une des plus importantes tient à la soif de renommée qui les anime. Leur fortune ayant été bâtie au prix d'une lutte sans merci avec la concurrence, ils sont soucieux de donner une image plus positive d'eux-mêmes une fois parvenus au faîte de leur puissance. Par le rôle qu'assument les Maisons de bienfaisance comme cœur spirituel, moral et social des communautés hua ch'iao, elles sont dans cette optique le plus sûr moyen d'engranger un fort capital symbolique. D'autant que, par le volet humanitaire de leurs activités, elles donnent des gages de respectabilité et peuvent de ce fait servir de paravent à des entreprises moins louables, voire permettre de blanchir de l'argent sale. Enfin, certains entrepreneurs ayant par le passé trempé dans des affaires criminelles ont, dans leur vieillesse, des états d'âme qu'ils cherchent à surmonter par la multiplication d'actes méritoires. Parfois, ces intentions, plutôt que de relever d'un froid calcul, trouvent dans un message onirique ou une maladie oraculaire une expression sublimée : le généreux philanthrope se pose alors en élu ayant obéi à un commandement divin. Cet ordre est consécutif à la visite d'un temple de la lignée de culte au rayonnement de laquelle il va ensuite contribuer. L'enquête révèle que les fondateurs de temples Ming, Chip, Pong Lai ou Têk K'a ont le plus souvent découvert ces mouvements par le biais de parents, d'amis ou de simples relations qui leur en ont vanté les mérites. Autre tendance notoire: les instigateurs de la construction de temples dans les régions périphériques du Nord-Est, du Nord et du Sud sont souvent des entrepreneurs fortunés arrivés sur place peu de temps auparavant, à partir de Bangkok ou de la Plaine centrale. Ils étaient déjà adeptes de Ming, Pong Lai ou Têk K'a avant d'émigrer en quête de nouvelles opportunités économiques et, en plus des motifs déjà évoqués, la création d'un temple est pour eux l'occasion de se camper dans le rôle valorisé de propagateur de la foi, tout en s'intégrant par le haut à la communauté hua ch'iao de la ville où ils viennent de s'établir. Ils renouent donc avec les motivations qui étaient celles de beaucoup de fondateurs de shan tang dans la Chine du XIX ${ }^{\mathrm{e}}$ siècle $^{20}$.

\section{Analogie de situation, pérennité des structures}

Les millions de Chinois qui émigrèrent dans les Nan yang, les «mers du Sud », entre les années 1860 et 1950, fuyaient la pauvreté endémique de leur patrie d'origine et notamment des provinces côtières du Guangdong, du Fujian et de

20 - Le rôle crucial joué par des nouveaux venus en quête de promotion sociale dans la création des shan tang de la Chine du XIX ${ }^{\mathrm{e}}$ siècle a été souligné par WiLliam T. Rowe, Hankow: Commerce and Society in a Chinese City, 1796-1889, Stanford, Stanford University Press, 1984, p. 248, ou, plus récemment, par Susan NaQuin, Peking. Temples and City Life, 1400-1900, Berkeley, University of California Press, 2000, p. 652. 
Hainan, dont ils provenaient massivement ${ }^{21}$. Néanmoins, pour la majorité d'entre eux, l'espoir d'un eldorado qui leur permettrait de faire fortune aisément se dissipa vite, tant la concurrence et les conditions de vie outre-mer étaient dures. Même dans un pays très favorable comme la Thaïlande, dont les souverains, jusqu'en 1910, voyaient dans l'afflux massif de coolies un moyen de développer l'économie nationale en toute indépendance, la misère de nombre d'entre eux resta notoire jusqu'à la Première Guerre mondiale. De gros contingents étaient exploités sans vergogne par des compatriotes auprès desquels ils s'étaient endettés pour payer leur voyage. Par ailleurs, jusqu'en 1920, les deux tiers des immigrés étaient de jeunes hommes ayant laissé en Chine femme et enfants: aux rythmes de travail effrenés et à l'asservissement s'ajoutait l'isolement psycho-affectif pour en pousser beaucoup vers des expédients, facteurs de paupérisation tels que le jeu, l'alcool et l'opium. William T. Skinner relève à cet égard la faiblesse du sentiment lignager outre-mer ${ }^{22}$ et le cynisme de l'État siamois qui, d'un côté, comptait sur la vertu des Chinois pour développer le commerce ou l'industrie, et, de l'autre, s'appuyait sur leurs vices pour accroître les revenus publics (via la création de taxes sur l'alcool, l'opium et les jeux de hasard). Le même auteur révèle que l'arrivée de 1,44 million de migrants dans le pays entre 1880 et 1917 fut contrebalancée en partie par le retour des deux tiers dans la mère patrie, l'ampleur de ce contre-flux étant l'indice d'un certain mal-être ${ }^{23}$.

À bien des égards donc, les immigrés de la première génération retrouvaient outre-mer la précarité qu'ils avaient laissée en Chine. Dans un premier temps, les sociétés secrètes, aux ramifications internationales, firent le lien entre le pays d'origine et les mers du Sud pour protéger leurs adhérents sur le mode du patronage. Selon John Mac Carthy, tous les Chinois de Siam étaient membres d'une triade à la fin du XIX ${ }^{\mathrm{e}}$ siècle $^{24}$. Néanmoins, ces organisations s'affrontaient avec violence et généraient de fortes tensions au sein de la communauté immigrée. Elles périclitèrent au début $\mathrm{du} \mathrm{Xx}^{\mathrm{e}}$ siècle sous la pression des autorités locales. Dans le même temps, émergèrent à Bangkok des associations dialectales (celle des locuteurs du hainanais en 1900, du hakka en 1909 ou du teochiu en 1918).

21 - Selon une estimation donnée par V. Purcell dans The Chinese in Southeast Asia..., op. cit., p. 3, un peu plus de 8,5 millions de Chinois vivaient dans la région en 1947, dont 2,5 millions, soit près d'un tiers, au Siam. La disette de 1846 qui frappa les provinces de Guangdong et du Fujian aurait provoqué la mort de 225000 personnes, d'après les chiffres fournis par CHEN TA, Chinese Migration, with Special Reference to Labor Conditions, Washington, Government Printing Office, 1923, p. 6. GarTh Alexander, Silent Invasion. The Chinese in Southeast Asia, Londres, Mac Donald, 1973, p. 10, estime pour sa part à 20 millions le nombre de victimes de la rébellion des T'ai ping qui embrasa le sud de la Chine entre 1850 et 1864 . Selon le même auteur, les combats qui se déroulèrent entre clans hakka et cantonais auraient provoqué la mort de 100000 personnes, tandis que la grande famine de 1877 aurait fait 10 millions de victimes supplémentaires.

22 - W. T. Skinner, A study of Chinese Community Leadership..., thèse citée, p. 312. 23 - Ibid., pp. 38 et 152. p. 3. 
Agissant cette fois de manière légale, elles créèrent des institutions à vocation sociale ou culturelle, bien qu'à usage exclusif de leurs adhérents.

En fait, c'est aux shan tang instaurées localement entre 1910 et 1949 qu'il revint de développer une action philanthropique de plus grande envergure et largement tournée vers la société locale. En effet, si Po Têk Hsiang T’üng eut d'abord pour vocation de soulager la misère des Chinois, à partir des années 1920, elle et ses suivantes dirigèrent la majeure partie de leurs opérations charitables vers les nécessiteux thaïs. Plusieurs raisons expliquent ce changement de cap. Tout d'abord, comme William T. Skinner l'a bien montré ${ }^{25}$, le réseau associatif chinois connut après 1918 un essor sans précédent en Thaïlande, qui était l'indice d'un embourgeoisement de la minorité hua ch'iao. Un écrémage sévère s'étant entre-temps produit, restaient sur place une majorité de Chinois relativement prospères. De plus, cette époque coïncide avec l'arrivée aux affaires de fils ou de petitsfils d'immigrés nés au Siam et mieux intégrés. Beaucoup d'entre eux étaient des héritiers qui pouvaient s'appuyer sur une fortune déjà constituée afin de satisfaire leur soif de prestige. Qui plus est, les self-made men ou leurs descendants restaient socialement proches des coolies, ce qui favorisait l'identification à leur sort. Enfin, et c'est peut-être la plus importante des raisons: la montée en puissance d'un ultra-nationalisme thaï dans les années 1920 et surtout 1930, et, corrélativement, l'essor d'un fort sentiment anti-chinois ne pouvaient qu'inciter les mieux lotis des hommes d'affaires à donner des gages ostentoires d'intégration. Ce qui les amena à reproduire, sans nécessairement le savoir, la stratégie d'insertion qui avait été celle des migrants internes ayant fait fortune dans les grandes cités marchandes chinoises de la fin du XIX ${ }^{\mathrm{e}}$ siècle.

Hormis ces recoupements conjoncturels, les shan tang de Thaillande rejoignent leurs homologues de la mère patrie sur bien des points, qu'ils aient trait à leurs logiques d'action ou à leur mode de fonctionnement. Comme jadis à Canton, à Shantou ou au Fujian, elles restent d'initiative privée et locale ${ }^{26}$. L'idée de leur création revient toujours à un ou plusieurs entrepreneurs de la ville où elles opèreront et non d'un quelconque centre de pouvoir, même si certaines s'inscrivent dans des lignées de culte, voire dans des mouvements sectaires d'envergure internationale.

Sur le plan des objectifs humanitaires, elles se dotent, comme en Chine pré-communiste, de moyens de secours qui suppléent les pouvoirs publics en intervenant dans les accidents individuels ou dans les catastrophes majeures. Ainsi Po Têk Hsiang T’üng disposait en l'an 2000 de cent ambulanciers organisés en

25 - W. T. Skinner, A Study of Chinese Community Leadership..., thèse citée, pp. 309-319. 26 - Sous l'impulsion de riches entrepreneurs locaux, la première shan tang ouvrit à Shanghai en 1804, et la ville comptait en 1850 quatre de ces institutions, selon les données fournies par MARK ELVIN, "The Gentry Democraty in Chinese Shanghai, 1905-1914 ", in J. Gray (éd.), Modern China's Search for a Political Form, Londres, Oxford University Press, 1060, pp. 42-43; à Pékin, les deux premières apparurent en 1845 et 1848, et leur nombre atteignit une douzaine dans la décennie suivant la révolte des Taiping; à Canton, la première ouvrit en 1871, ainsi que l'indique William T. Rowe, Hankow: Conflict and Community in a Chinese City, Stanford, Stanford University Press, 1989, p. 107. 
deux brigades et d'une vingtaine de véhicules d'intervention, le tout assurant $24 \mathrm{~h} / 24 \mathrm{~h}$ un service d'assistance rapide dans la capitale et sa banlieue; d'autre part, elle pouvait au besoin faire appel à mille sept cents bénévoles. Elle employait en tout 2200 salariés, dont 150 au centre de secours, 1300 à l'hôpital et 750 dans son centre universitaire ${ }^{27}$. Ajoutons encore que les shan tang thaïlandaises, à la manière de leurs archétypes chinois, prennent en charge l'inhumation des morts sans descendance, offrent des biens de première nécessité aux pauvres, soit ponctuellement, soit dans le cadre de fêtes calendaires chinoises $^{28}$, et financent des infrastructures dans les zones rurales déshéritées.

Du point de vue organisationnel cette fois, elles continuent d'être gouvernées par un directoire de riches entrepreneurs âgés ${ }^{29}$ et partiellement détachés des affaires. Parmi eux, un président est désigné selon le principe de la rotation et pour un mandat d'une durée variable. Cependant, au quotidien, leur administration est assurée par un directeur général, moins fortuné, mais plus expérimenté et cultivé, qui s'est avéré bon gestionnaire dans le secteur privé et manifeste aussi de grandes compétences sur le plan linguistique (bonne maîtrise du teochiu) et coutumier (connaissance de certains classiques et traités chinois).

Malgré tout, les différences sont notables par rapport à ce qui se faisait jadis. Tout d'abord, l'action philantropique des shan tang thaïlandaises ne présente plus le caractère confucéen de leurs homologues des Qing postérieurs : l'aide aux veuves vertueuses que développaient ces dernières a été abandonnée tout comme l'ingérence dans l'ordre public à travers le financement de milices. D'autre part, l'émigration a entraîné un fort resserrement institutionnel de l'activité religieuse et caritative. En Thaïlande, le système des guildes, si important dans la mère patrie d'avant 1949, n'a pas été transposé. De plus, les structures lignagères qui organisaient la Chine rurale ont connu un très fort fléchissement parmi les communautés hua ch'iao au caractère urbain ${ }^{30}$. Il faut aussi considérer la répression conduite

27 - Le statut de l'association dépendant d'abord et avant tout du nombre de ses salariés - c'est-à-dire, indirectement, de ses revenus -, Po Têk Hsiang T'üng reste à ce jour la plus puissante des associations caritatives chinoises du pays, même si, dans l'absolu, ses moyens humains paraissent moindres.

28 - La principale fête où elles procèdent à de larges distributions de nourriture est celle du Salut universel (si kou en teochiu), qui a lieu au cours du $7^{\mathrm{e}}$ mois lunaire. Pour l'occasion, toutes les shan tang de Thaïlande recueillent des fonds auprès de leurs sympathisants afin d'acheter des denrées de première nécessité (riz décortiqué, sauce de poisson fermentée, paquets de lessive, savonnettes, vêtements...), qui sont distribuées par lots aux indigents au terme de la fête. Tandis qu'une Maison de bienfaisance de province offre de la sorte une moyenne de 1500 à 2000 lots aux pauvres, la principale shan tang du pays, Pô Têk Hsiang 'T'üng, en distribue vingt à trente fois plus. En 2000, elle a ainsi offert 65000 lots, chacun d'une valeur de 300 bahts (l'équivalent de 8 dollars nord-américains) à une foule surtout composée de Thaïs.

29 - Dans un échantillon de vingt shan tang auprès desquelles j'ai enquêté entre 1993 et 2000, la moyenne d'âge des membres de ces directoires était de soixante-sept ans. 30 - Cette inflexion se signale par l'abandon presque général des temples lignagers où étaient conservées les tablettes ancestrales; voir BERNARD FORMOSO, Identités en regard. Destins chinois en milieu bouddhiste thä̈, Paris, Éditions de la MSH/CNRS Éditions, 2000. 
par les nationalistes locaux à l'encontre de l'hégémonisme économique chinois, qui entraîna l'interdiction de la plupart de leurs lieux de culte. Finalement, hormis les sanctuaires territoriaux généralement dénués de clergé, les seules institutions qui passèrent sans encombre l'épreuve de l'ultra-nationalisme thaï furent les associations caritatives dont l'intention affirmée était de redistribuer aux autochtones une part des richesses accumulées par les Chinois.

Pour ces divers motifs, les shan tang concentrèrent des services auparavant assumés par un plus grand nombre d'institutions, au point de devenir les principaux pôles religieux hua ch'iao de Thaïlande. Ce constat s'applique aussi à l'autre grande communauté chinoise d'Asie du Sud-Est, celle de Malaysia. Tan Chee-Beng écrit ainsi des shan tang de ce pays qu'elles peuvent être perçues comme des organisations dont l'activité caritative sert de prétexte à l'enseignement et à la pratique religieuse ${ }^{31}$.

En Thaïlande, les équipes de prêtres des Maisons de bienfaisance, qui ne se réclament ni des clergés bouddhistes ni des clergés taoïstes, mais des deux doctrines à la fois, prennent en charge les grands événements du cycle de vie des familles hua ch'iao à même de louer leurs services. Ils dominent en particulier l'organisation des rites funéraires: la veillée et la présentation des plats au défunt, le rituel kong têk (gong de) d'expression de la piété filiale, ou encore le placement géomantique des tombes. Ce sont les shan tang également qui supervisent, dans les villes où elles sont implantées, l'organisation des principales fêtes calendaires. De plus, et c'est là une nouveauté par rapport à leurs consœurs du pays d'origine, elles ont pour la plupart recours à l'écriture révélée ( $h u$ k'i en teochiu). Les séances présentent alors un caractère régulier ou occasionnel, mais ont toujours pour but de délivrer des ordres divins à l'association ou bien des oracles, des protections ou des médications aux individus victimes d'infortune.

Certains historiens ont montré que des activités médiumniques fondées sur des techniques oraculaires strictement identiques à celles aujourd'hui observables en Thaïlande se développèrent fortement dans la province du Guangdong dès la seconde moitié du XIX ${ }^{\mathrm{e}}$ siècle $^{32}$, leur premier objet étant de guérir diverses maladies en sollicitant auprès des divinités l'obtention de cures ou des intercessions spirituelles. Leur grande popularité découla notamment de leur aptitude à guérir en grand nombre les opiomanes. Or, comme à la même époque la consommation de drogue faisait des ravages parmi les coolies chinois au Siam, il est possible que l'écriture inspirée ait été introduite dans le pays pour servir les mêmes fins. Aujourd'hui cependant, cette tradition répond à une demande moins spécifique et ne se perpétue que dans le cadre des shan tang ou institutions affiliées ainsi que de quelques temples végétariens de Bangkok.

Dans ces associations religieuses, les médiums communiquent avec des divinités bien connues des Chinois, tels les Huit Immortels du panthéon taoïste, mais aussi, le plus souvent, avec des personnages légendaires de moindre envergure,

31 - Tan Chee-Beng, «The Religion of the Chinese... », art. cit., pp. 282-315.

32 - On pense notamment au Japonais Keijirō Marui, dont les recherches concernant l'histoire de l'écriture inspirée ont été traduites par GARY SEAMAN dans Temple Organization in a Chinese Village, Taipei, The Orient Cultural Service, 1978, pp. 24-28. 
dont l'hagiographie révèle qu'ils sont pour la plupart natifs des provinces de Guangdong et du Fujian d'où provenaient l'essentiel des Chinois de Thaïlande. On touche ici à une autre fonction des shan tang du pays. Leur activité religieuse, parce que centrée autour du culte de héros locaux (Da Feng, He Yeyun...) ou bien parce qu'elle naquit en pays teochiu (cas de De Jiao par exemple), concourt à la glorification d'une identité proprement régionale et maintient de la sorte un fort lien affectif avec une terre ancestrale plus immédiate que l'ensemble de la Chine, même si nombre de descendants d'immigrés n'ont pu, ou voulu, entretenir de relations avec leurs parents restés au pays. Autrement dit, ces organisations servent de vecteurs à l'entretien d'une communauté imaginée ${ }^{33}$, définie sur des bases dialectales (les Teochiu, tels qu'ils se démarquent des voisins Hokkien ou Hakka, par exemple). Pour les plus fortunés et disponibles des dévots, cette communauté imaginée peut d'ailleurs se concrétiser dans des images et un vécu immédiats grâce à des pèlerinages périodiques vers des temples mères ou des lieux de sépulture du pays d'origine ${ }^{34}$.

Un autre facteur de renforcement de cette identité teochiu dans le contexte particulier des Hua Ch'iao de Thaïlande tient à l'organisation périodique du hsiu kou ku. Par rapport à leurs compatriotes de Malaysia, de Singapour ou d'ailleurs, les Teochiu de Thaïlande tirent en effet une grande fierté de leur aptitude singulière à avoir perpétué et développé cette fête, dont la finalité explicite est l'acquisition des mérites, mais qui satisfait également d'autres visées. Ce cycle rituel, qui peut durer de sept à quatre-vingt-dix-neuf jours et engage des sommes considérables de l'ordre de dix millions de bahts en moyenne, procède d'une appropriation symbolique des ressources locales. En effet, de même que l'hégémonie économique des Chinois dans le pays les amène à employer une bonne part de la force de travail autochtone, ils étendent ce genre de rapports aux malemorts thaïs, qu'ils manipulent par des moyens rituels et géomantiques afin d'accroître leur prospérité en ce monde et obtenir de meilleures conditions de renaissance. De plus, en nettoyant périodiquement le paysage de tous les esprits maléfiques qui le hantent, les Hua Ch'iao donnent des gages de sens civique et donc d'intégration à l'ensemble national thaïlandais. Mais l'image qu'ils offrent alors d'eux-mêmes est ambiguë car, en manipulant sans réticences les ossements de malemorts devenus des esprits de forêt, esprits que les Thaïs redoutent, les Hua Ch'iao se trouvent identifiés d'une certaine manière aux forces maléfiques des confins. Sont ainsi renforcées les attitudes d'évitement et les frontières sociales qui démarquent les deux communautés.

Si les Maisons ou Autels de bienfaisance chinois se sont développés en Thaïlande dans des contextes de précarité socio-économique et d'instabilité politique

33 - On fait bien sûr ici référence à l'ouvrage de Benedict Anderson, Imagined Communities, Reflections on the Origin and Spread of Nationalism, Londres, Verso Books, 1983. 34 - Toutes les grandes shan tang du pays organisent chaque année des voyages collectifs vers Shantou, Xiamen et, plus largement, les provinces de Guangdong ou du Fujian, afin de visiter certains sites historiques auxquels sont étroitement identifiés les saints patrons du temple. Ces voyages font ensuite l'objet de reportages photographiques dans les rapports annuels de ces associations. 
proches de ceux qui avaient conduit à leur essor dans la Chine du XIX ${ }^{\mathrm{e}}$ siècle, une permutation importante au sein de la «structure de la conjoncture ${ }^{35}$ » se produisit. En effet, la prospérité atteinte après 1918 par de nombreux Hua Ch'iao et le sentiment anti-chinois des ultra-nationalistes siamois se conjuguèrent pour déplacer l'essentiel des actions caritatives des coolies vers la population autochtone.

Un autre constat qui intéresse aussi l'analyse historique du rapport entre structure et conjoncture tient aux adaptations qui marquèrent les finalités et l'organisation de ces associations. L'émigration hors de Chine se solda par des stigmates psychologiques dus au déracinement et par un fléchissement très sensible des idéologies patrilinéaire ou confucéenne, ainsi que par le non-transfert outre-mer des guildes. De plus, de nombreuses associations religieuses chinoises qui avaient fleuri au Siam avant la Première Guerre mondiale furent interdites par les nationalistes thaïs. Dans ce contexte, les Maisons de bienfaisance, parce qu'elles favorisaient en apparence les autochtones (quoique sur un mode typiquement hua ch'iao), étaient les seules à pouvoir être promues localement. Du coup, elles servirent en toute discrétion de dernier refuge à la tradition liturgique chinoise, assurant à l'usage exclusif des Hua Ch'iao tout à la fois la célébration des rites du cycle de vie, des fêtes calendaires et des activités médiumniques à vocation oraculaire (l'écriture révélée). L'origine teochiu d'une majorité d'immigrés et surtout d'animateurs de ces temples, en limitant la variabilité des formes d'expression religieuse en leur sein, favorisèrent ce processus, tout en soutenant, dans et par des formes de cultes spécifiques, une identité dialectale qui faisait le lien avec la terre des ancêtres. Enfin, et c'est sans doute le plus important, la domination des Teochiu dans les associations philanthropiques de Thaïlande aboutit à une structure de coopération inédite entre elles, qui n'a pas d'équivalent dans la Chine précommuniste (les shan tang y revêtaient une dimension strictement locale) et dans d'autres pays où les Hua Ch'iao sont établis.

Bernard Formoso

Université Paris X-Nanterre

35 - Selon le sens donné par Marshall SAHLins: «Un ensemble situationnel de relations qui se cristallise à partir des catégories culturelles à l'œuvre et des intérêts des acteurs» (Des îles dans l'histoire, Paris, Gallimard, 1989, p. 141, n. 11). 
Chi

Chi Kong

濟公

Chip T'ua

集壇

Ch'iah Hsiant'ong (Bai Yün)

張玄同

chías tuan

僑困

Hiang Sing Hsiang T'üng

玄辰善堂

bsiang t'ua (shan tan)

普壇

bsiang t'üng (shan tang)

善堂

bsiu kou ku

修孤骨

buki

扶乩

Lao Pehung

劉伯溫

Liu Ch'unwong

柳春芳

Ming T'ua

明埇

Po Têk Hsiang Tüng

報德善堂

Si Kak Hsiang Tüng

世覺善堂

sikou

施孤

Tai Hong (Da Feng)

大峰

tang $k$ 'i

童枝

Tchong Têk Hsiang T'üng

崇德善堂

Têk Tao Hsiang T'üng

道德善堂

Têk K'a

德教

Ya Hungsang

揚筠松

Wu Manghu

吳夢吾 